\title{
Case Report: \\ Trigger Wrist: A Case Series Study
}

Omid Mahmoudi Nasab (D), Mohammadamin Haghbin (D, Mohammad Reza Kooshesh (D), Farid Najd Mazhar (D)

1. Bone and Joint Reconstruction Research Center, Shafa Orthopedic Hospital, Iran University of Medical Sciences, Tehran, Iran.

\begin{tabular}{|l|l|}
\hline $\begin{array}{c}\text { Use your device toscan } \\
\text { and reat the article online }\end{array}$ & $\begin{array}{l}\text { Citation Mahmoudi Nasab O, Haghbin M, Kooshesh MR, Najd Mazhar F. Trigger Wrist: A Case Series Study. Journal of } \\
\text { Research in Orthopedic Science. 2019; 6(3):17-22. http://dx.doi.org/10.32598/JROSJ.6.3.17 }\end{array}$ \\
\hline
\end{tabular}

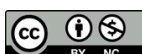

Article info:

Received: 29 Apr 2019

Revised: 05 May 2019

Accepted: 05 Jul 2019

Available Online: 01 Aug 2019

Keywords:

Trigger wrist, Etiology, Tumor, Clinical manifestation

\begin{abstract}
A B S T RA C T
Background: Trigger wrist is a relatively rare hand disorder that refers to the painful snapping and clicking or triggering at the wrist level during finger or wrist movements.

Objectives: In this study, we aimed at reporting a series of trigger wrist and discussing their etiology, as well as their clinical manifestation and outcomes.

Methods: In this retrospective study, the patients with the definitive diagnosis of trigger wrist were reviewed. Their demographics and clinical symptoms were extracted from their medical records. The symptoms of median neuropathy, including weakness or atrophy of the thenar muscles, persistent numbness, and paresthesia were recorded to investigate the association with Carpal Tunnel Syndrome (CTS).

Results: A total of seven patients with the Mean \pm SD age of $35.5 \pm 10.3$ years were included in the study. A benign mass was the etiology of trigger wrist in all cases, with giant cell tumor of flexor tendon sheet as the most common etiology (57.1\%). Trigger wrist was associated with CTS in three cases (42.8\%), in all of whom the tumor size was larger than the average. Pain and snapping with or without palpable mass were the main clinical symptoms of the patients that completely disappeared by surgical removal of the lesion. A mean 40.3 months follow-up of the patients was uneventful.

Conclusion: Benign neoplasms could be considered as the most common etiology of trigger wrist. Since many patients with trigger wrist might have CTS, too, the careful differentiation of these entities is necessary. Once accurately diagnosed, surgical intervention is entirely curative.
\end{abstract}

\section{Introduction}

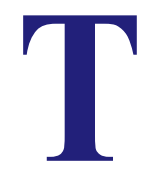

rigger finger is one of the most common upper limb disorders causing painful locking or clicking during finger motion. Although the inflammation and narrow- ing the space of A1 pulley is associated with trigger finger disorder, its exact etiology remains unknown [1]. In contrast to the trigger finger, the "trigger wrist" is a relatively rare condition that refers to the painful clicking and snapping, as well as catching sensation at the wrist level during finger or wrist movements. It is

\section{* Corresponding Author:}

Omid Mahmoudi Nasab, PhD.

Address: Bone and Joint Reconstruction Research Center, Shafa Orthopedic Hospital, Iran University of Medical Sciences, Tehran, Iran.

Phone: +98 (21) 66948785

E-mail: o.mayahi@gmail.com 
also known as "snapping wrist" or "clicking wrist" [2]. Trigger wrist usually occurs in adults, and it is scarce in children or teenagers [3].

Carpal tunnel provides limited space for its contents such as flexor tendons and median nerve. The chronic compression of the median nerve within the carpal tunnel results in an entrapment neuropathy called Carpal Tunnel Syndrome (CTS) [4]. The trigger wrist is attributed to pathologies under or around the transverse carpal ligament and flexor tendons. An anomalous muscle crossing the carpal tunnel, a tumor mass within the carpal tunnel, or a combination of both entities are the most common etiologies of the trigger wrist [5]. Since these etiologies might also narrow the carpal tunnel, the median nerve might be compressed, as well. For this reason, patients with trigger wrist are frequently presented with CTS [6].

To date, the majority of studies concerning trigger wrist are presented as case reports, and only a few case series are available on this subject in the literature. In this study, we aimed at reporting a series of trigger wrist and discussing their etiology, as well as their clinical manifestation and outcome.

\section{Case Presentation}

The Ethics Committee of our institute approved the present study, and the patients provided informed consent to use their medical data for publication. In the present retrospective study, we reviewed the electronic files of the patients with trigger wrist, who were referred to our center from 2008 to 2018 .

The clinical and demographic characteristics of the patients were extracted from their medical records. The symptoms of median neuropathy, including weakness or atrophy of the thenar muscles, persistent numbness, and paresthesia, were evaluated as a part of the diagnostic procedure. The electrodiagnostic study was performed for patients with the clinical symptoms of CTS. The condition was assessed by plain radiographs and Magnetic Resonance Imaging (MRI). If a mass caused the disease, the mass would be extracted and sent to the pathology department for definitive diagnosis.

\section{Treatment approach}

A fellowship-trained hand surgeon performed all operations. Under general anesthesia and tourniquet control, the surgeon initially explored the carpal tunnel and released the flexor retinaculum. After the exploration of the flexor tendons, the underlying mass was identified.
Subsequently, the surgeon excised the mass and sent for histologic examinations. Also, in patients with CTS symptoms, the carpal tunnel was released.

A dorsal short-arm splint was used for two weeks after the operation allowing for unrestricted finger range of motion. The first follow-up visit of the patients was two weeks after the operation. In this session, the sutures and the splint were removed. The patients were visited every month for the evaluation of the stiffness, every three months for the evaluation of potential complications, and every six months for the survey of recurrence.

A total of seven patients were diagnosed with trigger wrist during the study period and included in this survey. The study population included one (14.3\%) woman and six (85.7\%) men with the Mean \pm SD age of $35.5 \pm 10.3$ years (range: $20-51$ years). The disease involved the dominant hand in five $(71.4 \%)$ cases and non-dominant in two $(28.6 \%)$ cases. The location of involvement was proximal to the carpal tunnel in six $(85.7 \%)$ cases and distal to the carpal tunnel in one $(14.3 \%)$ case. In all cases, a mass was identified in the MRI investigation of the carpal tunnel (Figure 1).

The Mean \pm SD size of the lesions at their largest diameter was $14 \pm 5.5 \mathrm{~mm}$ (range: $10-23 \mathrm{~mm}$ ). The main symptoms were pain and snapping. In one case (Case No. 6), a mass was palpable at the affected region. Median nerve neuropathy symptoms without thenar muscle atrophy were detected in three $(42.8 \%)$ cases. Mild to moderate CTS was present in the preoperative electromyography of these three patients. The mean size of the mass was considerably more in these patients, as well (19.3mm vs. $10 \mathrm{~mm})$. Table 1 presents the clinical, demographic, and pathologic characteristics of the patients.

Based on the report of pathology, the etiology of trigger wrist was a benign mass in all cases. In this respect, the most common etiology was Giant Cell Tumor (GCT) of the tendon sheet that was seen in four $(57.1 \%)$ cases. The etiology was one ganglion cyst, one lipoma, and one fibroma in the three remaining cases (Figure 2).

After the excision of the mass, the symptoms completely disappeared in all patients. A 3-month followup of the patients was event-free. At a Mean \pm SD follow-up of 40.3 \pm 13.7 months (range: $24-60$ months), no recurrence was observed in any case. No other complications were also recorded. 

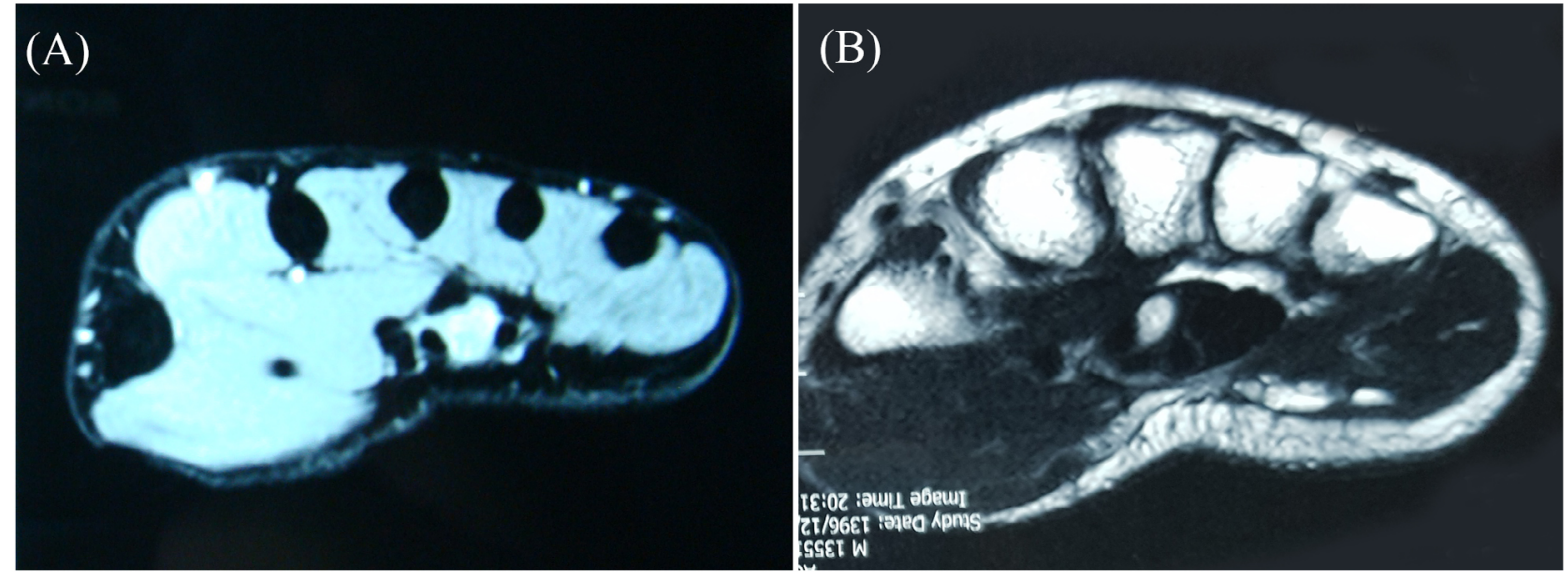

Figure 1. Axial MRI of wrist showing a soft-tissue mass proximal to the carpal tunnel: A: Case 1; B: Case 5 Orthopedic Science

\section{Discussion}

Trigger wrist is an infrequent condition and, therefore, only a small number of case series concerning the trigger wrist are available in the literature. In this study, we retrospectively reviewed the clinic-demographic char- acteristics, etiology, and outcome of patients, who were diagnosed with trigger wrist during the study period. In this regard, a benign mass was the etiology of trigger wrist in all cases of this series, with GCT of flexor tendon sheet as the most common etiology (57.1\%). The mass was located proximal to the carpal tunnel in the
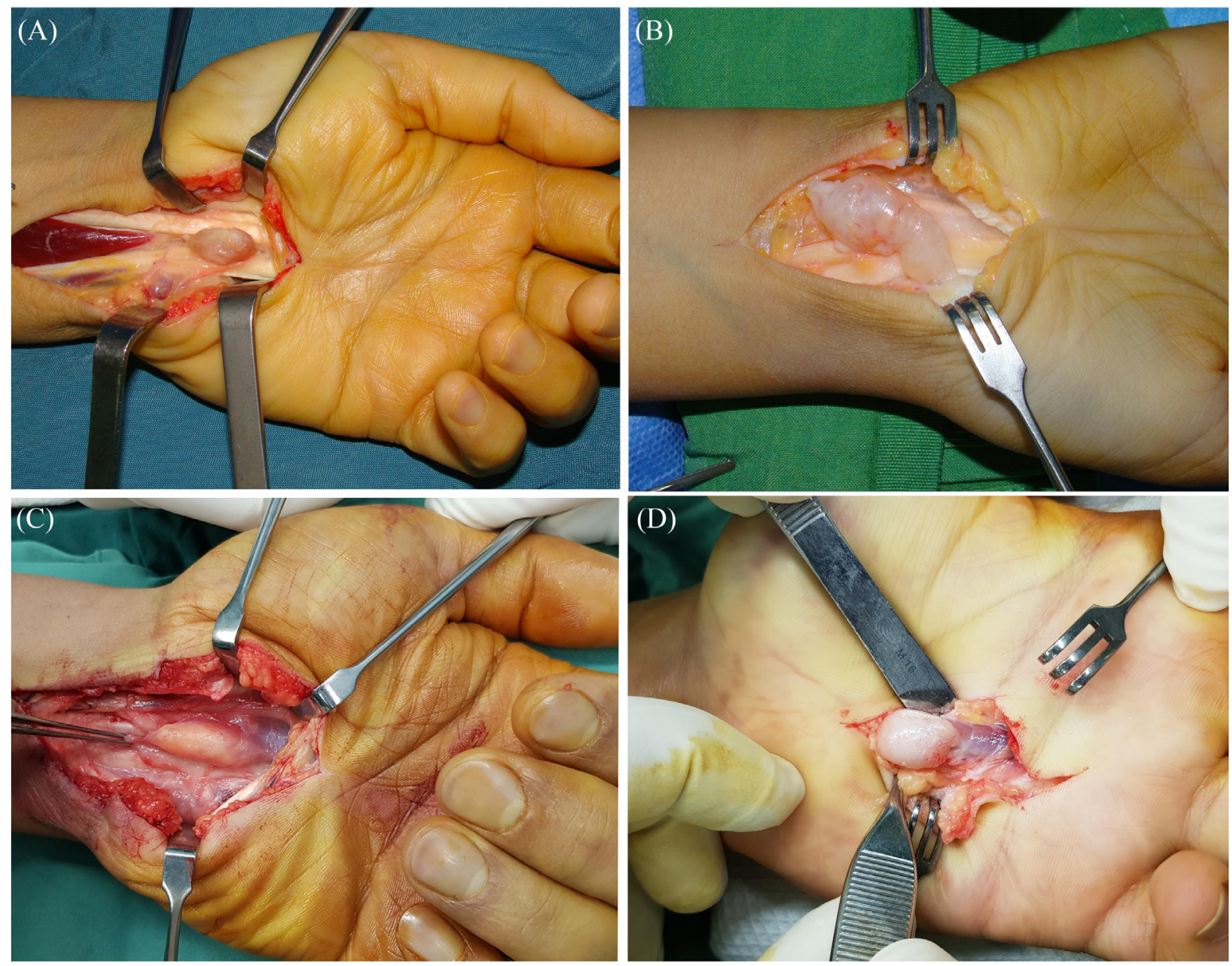

Orthopedic Science

Figure 2. Intraoperative images showing a mass causing the trigger wrist: A: Case 1; B: Case 3; C: Case 5; D: Case 6 
Table 1. The clinical, demographic, and pathologic characteristics of patients with trigger wrist

\begin{tabular}{|c|c|c|c|c|c|c|c|c|c|}
\hline ID & $\begin{array}{c}\text { Age } \\
\text { (years) }\end{array}$ & Gender & $\begin{array}{c}\text { Hand } \\
\text { dominancy }\end{array}$ & Location & Symptoms & Size $(\mathrm{mm})$ & Etiology & CTS & $\begin{array}{l}\text { Follow-up } \\
\text { (Months) }\end{array}$ \\
\hline 1 & 45 & Female & Non-dominant & Proximal to tunnel & Pain \& snapping & $10 \times 8 \times 5$ & $\mathrm{GCT}$ & No & 54 \\
\hline 2 & 30 & Male & Dominant & Proximal to tunnel & snapping & $10 \times 10 \times 9$ & $\mathrm{GCT}$ & No & 60 \\
\hline 3 & 36 & Male & Dominant & Proximal to tunnel & Pain \& snapping & $23 \times 15 \times 10$ & Ganglion cyst & Yes & 36 \\
\hline 4 & 51 & Male & Dominant & Proximal to tunnel & Pain \& snapping & $20 \times 15 \times 10$ & $\mathrm{GCT}$ & Yes & 24 \\
\hline 5 & 37 & Male & Dominant & Proximal to tunnel & Pain \& snapping & $15 \times 10 \times 10$ & Lipoma & Yes & 30 \\
\hline 6 & 20 & Male & Dominant & Distal to tunnel & Mass \& snapping & $10 \times 10 \times 8$ & Fibroma & No & 48 \\
\hline 7 & 30 & Male & Non-dominant & Proximal to tunnel & snapping & $10 \times 10 \times 10$ & $\mathrm{GCT}$ & No & 30 \\
\hline
\end{tabular}

${ }^{*}$ CTS: Carpal tunnel syndrome

Orthopedic Science

*GCT: Giant cell tumor

majority of cases $(85.7 \%)$. Male gender and dominant hand were more involved, as well $(85.7 \%$ and $71.4 \%$, respectively). Trigger wrist was associated with CTS in three cases, in all of whom the tumor size was larger than the average. Pain and snapping with or without palpable mass were the main clinical symptoms of the patients. In all patients, the symptoms completely disappeared after the resection of the mass.

Although Suematsu et al. categorized the etiology of trigger wrist in three groups; tumor, anomalous muscle, or both, other pathologies have been recently described for trigger wrist [5]. Wang et al. reported a case of trigger wrist, in which the degenerative changes of the flexor tendon sheath was the underlying cause [7]. Wakasugi et al. reported a case of trigger wrist associated with CTS caused by localized amyloidosis [8]. In the report of Matsui et al., the trigger wrist was caused by the avascular necrosis of the capitate [9]. However, these etiologies are not as frequent as the etiology described by Suematsu et al.

Ogino et al. reported a series of four patients with trigger wrist. The etiology of disorder included rheumatoid nodule (one case), lipofibroma (one case), GCT of the flexor tendon sheath (one case), and partial laceration of the flexor digitorum superficialis tendon (one case) [10].

Park et al. reviewed the clinical presentations and outcome of operation in 15 trigger wrists with multiple etiologies. The etiology of disorder was severe tenosynovitis of the flexor tendon in four cases, an anomalous muscle of the flexor digitorum superficialis in five cases, fibroma of the flexor tendon sheath in two cases, and a rheumatoid nodule in one case. A combination of factors was the underlying cause of disorder in the remain- ing cases. A mild to moderate median nerve neuropathy without the atrophy of thenar muscle was seen in all patients of their series [2]. Unlike the study of Park et al., no anomalous muscle belly was detected as the cause of trigger wrist in the current series. Besides, the median neuropathy was seen only in patients with a mass sized larger than average.

Accurate clinical, physical, and radiologic examinations are necessary to prevent improper and time-wasting practice for the affected patients. Inappropriate treatment might result in conditions, which require more extensive surgeries such as the adhesion of flexor tendons, severe tenosynovitis, or advanced CTS. Trigger finger and CTS are the main differential diagnoses of trigger wrist. A vague pain on palm, difficulties in fingers' motion, and no point tenderness on A1 pulley might suggest trigger wrist [10]. If CTS is coupled with a trigger finger, triggering symptom should be examined at wrist level [11].

Since many patients with trigger wrist are accompanied by CTS, median neuropathy might be the chief complaint of the patients. In these cases, the evaluation of crepitus of intracarpal tunnel might help find an intracarpal mass. If crepitus is noticed through a slight pressure over the transverse carpal ligament, further evaluation such as MRI is warranted to differentiate the nature of tunnel-occupying lesion causing the trigger wrist [6]. In some cases of trigger wrist, the characteristic snapping or triggering around wrist might be absent. The clinical differentiation of trigger wrist is difficult in these cases [6]. Ultrasound imaging can provide a dynamic picture for the tendon triggering and could be helpful if trigger finger at the wrist is suspected [7]. 
Once the diagnosis is made, surgical management is the treatment of choice. Carpal tunnel release is generally performed for cases with associated CTS syndrome [12-14]. The carpal tunnel was released in all cases of the present series that were associated with CTS. The disappearance of triggering has been demonstrated in all the reports after surgical treatment $[2,6,10]$. Similarly, the symptoms were resolved in all patients of the present series following the surgical intervention.

Trigger wrist is a rare entity, and, thus, more awareness is needed regarding its accurate diagnosis and treatment. Benign neoplasms could be considered the most common etiology of trigger wrist. As many patients might also have CTS, the careful differentiation of trigger wrist from CTS is necessary. Once accurately diagnosed, surgical intervention is required for complete treatment.

\section{Ethical Considerations}

\section{Compliance with ethical guidelines}

This study was approved by the Ethics Committee of Bone and Joint Reconstruction Research Center, Iran University of Medical Sciences, Tehran.

\section{Funding}

This research did not receive any specific grant from funding agencies in the public, commercial, or not-forprofit sectors.

\section{Authors' contributions}

All authors contributed in preparing this article.

\section{Conflict of interest}

The authors declared no conflict of interest.

\section{References}

[1] Makkouk AH, Oetgen ME, Swigart CR, Dodds SD. Trigger finger: Etiology, evaluation, and treatment. Curr Rev Musculoskelet Med. 2008; 1(2):92-6. [DOI:10.1007/s12178-0079012-1] [PMID] [PMCID]

[2] Park IJ, Lee YM, Kim HM, Lee JY, Roh YT, Park CK, et al Multiple etiologies of trigger wrist. J Plast Reconstr Aesthet Surg. 2016; 69(3):335-40. [DOI:10.1016/j.bjps.2015.10.030] [PMID]
[3] Al-Qattan MM, Elshamma NA, Alqabbani A. Trigger wrist and carpal tunnel syndrome caused by a flexor tendon-related ganglion in a teenager: A case report. Int J Surg Case Rep. 2017; 30:86-8. [DOI:10.1016/j.ijscr.2016.11.051] [PMID] [PMCID]

[4] Marquardt TL, Evans PJ, Seitz Jr, Li ZM. Carpal arch and median nerve changes during radioulnar wrist compression in carpal tunnel syndrome patients. J Orthop Res. 2016; 34(7):1234-40. [DOI:10.1002/jor.23126] [PMID] [PMCID]

[5] Suematsu N, Hirayama T, Takemitsu Y. Trigger wrist caused by a giant cell tumour of tendon sheath. J Hand Surg. 1985; 10(1):121-3. [DOI:10.1016/S0266-7681(85)80038-3]

[6] Park IJ, Lee YM, Rhee SK, Song SW, Kim Hm, Choi KB. Trigger wrist. Clin Orthop Surg. 2015; 7(4):523-6. [DOI:10.4055/ cios.2015.7.4.523] [PMID] [PMCID]

[7] Wang Q, Jackson JKH, Png MA. Trigger finger at wrist caused by degenerative changes of the flexor tendon sheath and carpal tunnel syndrome: A case report. Case Rep Plast Surg Hand Surg. 2017; 4(1):48-51. [DOI:10.1080/23320885.20 17.1331135] [PMID] [PMCID]

[8] Wakasugi T, Shirasaka R, Kawauchi T, Fujita K, Okawa A. Carpal tunnel syndrome and trigger wrist caused by localized amyloidosis: A case report. J Hand Surg Asian Pac Vol. 2017; 22(04):508-11. [DOI:10.1142/S0218810417720340] [PMID]

[9] Matsui Y, Kawamura D, Kida H, Hatanaka KC, Iwasaki $\mathrm{N}$. Trigger wrist caused by avascular necrosis of the capitate: A case report. BMC Musculoskelet Disord. 2018; 19:90. [DOI:10.1186/s12891-018-2010-1] [PMID] [PMCID]

[10] Ogino T, Kato H, Ohshio I. Trigger wrist induced by finger movement. Pathogenesis and differential diagnosis. Handchir Mikrochir Plast Chir.1994; 26(1):3-6. [PMID]

[11] Aghasi M, Rzetelny V, Axer A. The flexor digitorum superficialis as a cause of bilateral carpal-tunnel syndrome and trigger wrist: A case report. J Bone Joint Surg Am. 1980; 62(1):134-5. [DOI:10.2106/00004623-198062010-00022]

[12] Giannikas D, Karabasi A, Dimakopoulos P. Trigger. J Hand Surg Eur Vol. 2007; 32(2):214-6. [DOI:10.1016/J. JHSB.2006.10.016] [PMID]

[13] Shimizu A, Ikeda M, Kobayashi Y, Saito I, Mochida J. Carpal tunnel syndrome with wrist trigger caused by hypertrophied lumbrical muscle and tenosynovitis. Case Rep Orthop. 2015; 2015(705237):1-3. [DOI:10.1155/2015/705237] [PMID] [PMCID]

[14] Berlund P, Kalamaras M. A case report of trigger wrist associated with carpal tunnel syndrome caused by an intramuscular lipoma. Hand Surg. 2014; 19(02):237-9. [DOI:10.1142/ S0218810414720174] [PMID] 
This Page Intentionally Left Blank 